\title{
Unexpected difficult intubation. is it a solvable problem? reflections for a safe approach
}

\begin{abstract}
Approximately $4 \%$ of tracheal intubations are unexpectedly difficult. In the world annually million people have general anesthesia. In terms of numbers it is perhaps one of the most critical moments of the entire anesthetic procedure. Sophisticated tools, and scientific studies in opposition creates a sometimes very crowded and confusing area in which anesthesiologists, particularly the younger ones, find difficulties when they encounter so many options and are not sure which to choose.

If you want a solution to the unexpected difficulties you must find a path reliable, simple and safe by identifying when and how to act and to insert into the training programs of the schools of anesthesia as a basic procedure. The review of the literature, analysis and revision of the tracheal intubation procedure for general anesthesia in elective surgery lead to the conclusion that a combined approach using tools that display the glottic region and instruments that also display the trachea allows the tube to be introduced and placed under vision and resolves the majority of difficult cases.
\end{abstract}

Volume I Issue 5 - 2014

Francesco Sgalambro

Department Anesthesia and Intensive Care, Oncology Institute of the Mediterranean, Italy

Correspondence: Francesco Sgalambro, Director Department Anesthesia and Intensive Care, Oncological Institute of the Mediterranean, via Penninazzo 7 Viagrande-Catania, Italy, Tel 39095-7895000 -609, 39-368-3। I2427, Email fr.sgalambro@tiscali.it

Received: October 31, 2014 | Published: November 13, 2014

\section{Opinion}

Approximately 230 million major surgical procedures are performed annually around the world as reported by The Lancet in the editorial 'Surgical Outcomes and Opportunities'. ${ }^{1}$ In US everyday about 60,000 people have general anesthesia, ${ }^{2}$ in UK 2.9 million annually, ${ }^{3}$ Approximately $4 \%$ of tracheal intubations are unexpectedly difficult. In terms of numbers it is perhaps one of the most critical moments of the entire anesthetic procedure. Data reported in the literature also indicate that $30 \%$ of the incidents totally attributable to anesthesia are due to difficulties in airway management. ${ }^{4}$ The scientific community has long focused on this problem by establishing study groups or by creating scientific societies dedicated to airway management. Industry has marketed dedicated devices as a result of constant advances in imaging technology. The most prestigious journals are aware of the importance of the issue and have provided more space for discussion and debate.

The number of guidelines, sophisticated tools, and scientific studies in opposition creates a sometimes very crowded and confusing area in which anesthesiologists, particularly the younger ones, find difficulties when they encounter so many options and are not sure which to choose. For expected difficulties Intubation steps are well defined. The criteria of predictability and the opportunity to study with CT 3-D and virtual laryngotracheoscopy in cases of subversion of anatomical structures allows programming of the intervention strategy. ${ }^{5,6}$ In the case of unanticipated difficulties this is not. The algorithms for the guidelines indicate the main path but leave ample room for a choice of techniques.

If you want a solution to the unexpected difficulties you must find a path reliable, simple and safe to insert into the training programs of the schools of anesthesia as a basic procedure. The approach to the problem must be reversed by analyzing and reviewing the foundations of tracheal intubation procedures for general anesthesia and identifying the critical times, devices, and techniques. When and how to act must be determined. The phases of tracheal intubation aimed at general anesthesia are three fold:

i. Pharmacological

\section{ii. Visualization of the glottis and vocal cords}

\section{iii. Introducing the tracheal tube}

The pharmacological phase is preparatory to ease ventilation and intubation. A deep level of anesthesia and muscle relaxation facilitates both ventilation and intubation. ${ }^{7,8}$ The pharmacological sequence must provide verification of ventilability prior to muscle relaxant administration. ${ }^{9}$ The visualization phase can be performed with a direct laryngoscope (such as a McIntosh) or with a video laryngoscope (VLS) in which an image is captured near the glottis and transmitted to video. Tube introduction takes place in both cases according to a direct axis with VLS is necessary to use routine spindles or other systems to address the tube.

The vocal cords may be clearly seen but may not be easy tube introduction However, when second standard procedure, oxygenated the patient, induced anesthesia, verified ventilability with a face mask, and administered the muscle relaxant, is carried laryngoscopy and, with both the traditional system or with VLS, there is located opposite to an unexpected Cormack 3 or 4 the spiral of danger begins, as follows: three attempts, looking for a more experienced colleague, other attempts, using spindles and introducers, switching to another system; begin secretions, bleeding or a decrease in oxygen saturation, we resort to additional ventilation, anesthesia becomes superficial , face mask ventilation becomes difficult, and the danger zone is entered.

This situation has three possible outcomes:

\section{Intubation}

\section{Not intubated but ventilated}

\section{Not intubated and non-ventilated}

In the first case the problem is solved. In the second case ventilation is continued with a face mask or with principals extraglottic and whether intubation is needed for surgery, the patient wakes up and is programmed in an awake intubation with a flexible bronchoscope (FOB). The third case requires tracheotomy. A dangerous situation should be avoided because at this level all interventions are 
difficult and there is no assurance of success. All optical devices e.g., Videolaryngoscopes, Bonfils, FOB, Air Traq, Optical Stylets, etc., in an environment polluted with blood and secretions and in an emergency scenario are used with difficulty, and procedures are successful only when performed by experts.

To maintain a workspace clean and free of blood and prevent adverse events during laryngoscopy when a Cormack score of $3-4$, logic dictates that we must refrain from blind attempts and go directly to the optical instrument. Do not make any blind attempts and stopping at the seconde step of the procedure laryngoscopy: this is when. But how? Among all available techniques, that with the greatest likelihood of successful intubation must be selected. Approaches using individual instruments (VLS, Air-Traq, Bonfils etc.) have success rates of $97-98 \%{ }^{10-12}$ A combined approach using tools that display the glottic region and instruments that also display the trachea allows the tube to be introduced and placed under vision and resolves the majority of difficult cases. ${ }^{13-16}$

A combined approach must be chosen. These combinations of McIntosch + Fob, ${ }^{15}$ VLS + FOB,${ }^{13-16}$ AirTraq + FOB,${ }^{17}$ VLS + Bonfils, ${ }^{14-18}$ McIntosch + Optical Stylet ${ }^{19}$ have been reported to be successful under difficult conditions, which confirms that all stages of intubation should be performed under vision. The intervention must select one of the combined approaches that has the greatest likelihood of success, is the most secure, and is easy to perform, facilitating secure ventilation in the event of failure (face mask or extraglottic tools) and allowing patient to awaken. Macintosh laryngoscope and VLS are used for visualizing the glottis but VLS are the preferred allows an anatomical view that facilitates guiding the optical instrument used to introduce the tracheal tube. The instrument used to introduce the tracheal tube can be rigid or flexible.

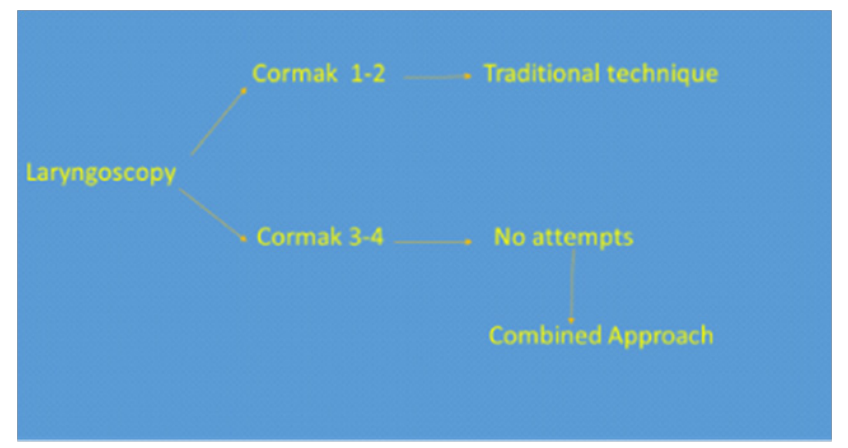

Figure I Algorithm for easy intubation.

The literature describes serious injuries due to the use of rigid instruments. Thus, flexible fiber optic instruments should be used. The FOB is considered the gold standard for tracheal intubation ${ }^{20}$ but under anesthesia with muscle relaxation is used with difficulty for the collapse of the anatomical planes. The Mc Intosh laryngoscope or the video laryngoscope create space for easy passage of the FOB. ${ }^{15} \mathrm{~A}$ VLS has the advantage of displaying the entire procedure and allowing video recording, unlike the Mcintosh laryngoscope. Therefore, a combined approach with VLS or Mcintosh and FOB is the gold standard for an unexpectedly difficult intubation (Figure 1).

However, the anesthetist considers the FOB as a tool of the bronchoscopist, has great confidence with video laryngoscopes and laryngoscopes, but its usefulness stops in the area of the laryngeal aditus. The glottis are considered the impassable limit of vision. Standardization of the combined approach technique with adaptation to standard manual maneuvers of the anesthesiologist can overcome this issue and enable a steep learning curve. The educational program in anesthesia should focus on the flexible bronchoscope, freeing it from the marginal role of an instrument reserved for a few. Use of the flexible bronchoscope for tracheal intubation in anesthesia should be part of basic education, together with the combined approach. Only starting from the revision of the procedure according to the principle that to succeed we must first do no harm, and routine use of the combined approach according to the algorithm for easy intubation, will overcome the issues associated with unexpectedly difficult intubations in elective surgery. This would represent a radical change in the approach to what is considered to be the basic procedure for anesthetist.

\section{Funding details}

None.

\section{Acknowledgments}

None.

\section{Conflicts of interests}

Authors declare that there is no conflict of interest.

\section{References}

1. Surgical Outcomes and Opportunities. Lancet. 2012;380:9847.

2. Waking Up to Anesthesia-Learn More Before You Go Under, NHINews in Health, 2011.

3. Cook TM, Woodall N, Frerk C. Major complications of airway management in the UK: results of the Fourth National Audit Project of the Royal College of Anaesthetists and the Difficult Airway Society. Part 1: anaesthesia. Br J Anaesth. 2011;106(5):617-631.

4. Schmidt U, Eikermann M. Organizational Aspects of Difficult Airway Management: Think Globally, Act Locally. Anesthesiology. 2011;114(1):3-6.

5. Sgalambro F, Sanfilippo F, Santonocito C, et al. Virtual laryngoscopy and combined laryngoscopic bronchoscopic approach for safe management of obstructive upper airway lesions. Br J Anaesth. 2014;113(2):304-306.

6. Agarwal S, Bates WB, Castresana MR. Images in Anesthesiology: Endoluminal Computed Tomography: A Novel Technology for Assessment of Large Airway Pathologies. Anesthesiology. 2014;121(1):170.

7. Ikeda A, Isono S, Sato Y, et al. Effects of Muscle Relaxants on Mask Ventilation in Anesthetized Persons with Normal Upper Airway Anatomy. Anesthesiology. 2012;117(3):487-493.

8. Warters RD, Szabo TA, Spinale FG, et al. The effect of neuromuscular blockade on mask ventilation. Anaesthesia. 2011;66:163-167.

9. Pandit JJ. Checking the ability to mask ventilate before administering long-acting neuromuscular blocking drugs. Anaesthesia. 2011;66(6):520-522.

10. Aziz MF, Healy D, Kheterpal S, et al. Routine clinical practice effectiveness of the Glide scope in difficult airway management: an analysis of 2,004 Glide scope intubations, complications, and failures from two institutions. Anesthesiology. 2011;114(1):34-41.

11. Amathieu R, Combes X, Abdi W, et al. An algorithm for difficult airway management, modified for modern optical devices (Airtraq laryngoscope; LMA CTrach ${ }^{\mathrm{TM}}$ ):A 2-year prospective validation in patients for elective abdominal, gynecologic, and thyroid surgery. Anesthesiology. 2011;114(1):25-33. 
12. Falcetta S1, Pecora L, Orsetti G, et al. The Bonfils fiberscope: a clinical evaluation of its learning curve and efficacy in difficult airway management. Minerva Anestesiol. 2012;78(2):176-184.

13. Lenhardt R, Burkhart MT, Brock GN, et al. Laryngoscope-Assisted Flexible Tracheoscope Intubation Feasible for Patients with Predicted Difficult Airway? A Prospective, Randomized Clinical Trial. Anesth Analg. 2014;118(6):1259-1265.

14. Maybauer MO, Maier S, Thierbach AR. An unexpected difficult intubation. Bonfilsrigid Fiberscope. Anaesthesist. 2005;54(1):35-40.

15. Sgalambro F, Denaro A, Guglielmo M, et al. An Algorithm For Easy Intubation. Combined Use Of The MacIntosh Laryngoscope An Flexible Bronchoscope in Unexpected Difficult Intubation. Acta Medica Mediterranea. 2013;29:437

16. Sharma D, Kim LJ, Ghodke B. Successful airway management with combined use of Glidescope videolaryngoscope and fiber optic bronchoscope in a patient with Cowden syndrome. Anesthesiology. 2010;113(1):253-255.
17. Yuan YJ, Xue FS, Liao X, et al. Facilitating combined use of an Airtraq ${ }^{\circledR}$ optical laryngoscope and a fiber optic bronchoscope in patients with a difficult airway. Can J Anaesth. 2011;58(6):584-585.

18. Liao X, Xue FS, Cheng Y, et al. Rescue intubation by combined use of video laryngoscopy and Bonfils fiberscope in patients with difficult airway. Saudi Med J. 2013;34(9):970-971.

19. Lin N, Li M, Shi S, et al. ShikaniTM Seeing Optical Stylet-aided tracheal intubation in patients with a large epiglottic cyst. Chin Med J Engl. 2011;124(17):2795-2798.

20. Asai T. Video laryngoscopes: do they truly have roles in difficult airways? Anesthesiology. 2012;116(3):515-517. 